\title{
Realization of hydrodynamic experiments on quasi-2D liquid crystal films in microgravity
}

\author{
Noel A. Clark ${ }^{1}$, Alexey Eremin ${ }^{2}$, Matthew A. Glaser ${ }^{1}$, Nancy Hall ${ }^{3}$, Kirsten \\ Harth $^{2}$, Christoph Klopp ${ }^{2}$, Joseph E. Maclennan ${ }^{1}$, Cheol S. Park ${ }^{1}$, Ralf \\ Stannarius ${ }^{2}$, Padetha Tin ${ }^{4}$, William N. Thurmes ${ }^{5}$, Torsten Trittel ${ }^{2}$ \\ ${ }^{1}$ Department of Physics, University of Colorado Boulder, Boulder, CO, USA \\ ${ }^{2}$ Institute of Experimental Physics, Otto von Guericke University, Magdeburg, Germany \\ ${ }^{3}$ NASA Glenn Research Center, Cleveland, OH, USA \\ 4 Universities Space Research Association, Cleveland, OH, USA \\ ${ }^{5}$ Miyota Development Center of America, Longmont, CO, USA
}

\begin{abstract}
Freely suspended films of smectic liquid crystals are unique examples of quasi two-dimensional fluids. Mechanically stable and with quantized thickness of the order of only a few molecular layers, smectic films are ideal systems for studying fundamental fluid physics, such as collective molecular ordering, defect and fluctuation phenomena, hydrodynamics, and nonequilibrium behavior in two dimensions (2D), including serving as models of complex biological membranes. Smectic films can be drawn across openings in planar supports resulting in thin, meniscus-bounded membranes, and can also be prepared as bubbles, either supported on an inflation tube or floating freely. The quantized layering renders smectic films uniquely useful in 2D fluid physics. The OASIS team has pursued a variety of ground-based and microgravity applications of thin liquid crystal films to fluid structure and hydrodynamic problems in 2D and quasi-2D systems. Parabolic flights and sounding rocket experiments were carried out in order to explore the shape evolution of free floating smectic bubbles, and to probe Marangoni effects in flat films. The dynamics of emulsions of smectic islands (thicker regions on thin background films) and of microdroplet inclusions in spherical films, as well as thermocapillary effects, were studied over extended periods within the OASIS (Observation and Analysis of Smectic Islands in Space) project on the International Space Station. We summarize the technical details of the OASIS hardware and give preliminary examples of key observations.
\end{abstract}

Keywords: liquid crystal, smectic, microgravity, 2D hydrodynamics PACS: 05.45.-A, 45.50.-j, 81.70.Ha, 83.10.Pp

\footnotetext{
*Corresponding authors: R.S., ralf.stannarius@ovgu.de, J.E.M., jem@colorado.edu
} 


\section{Introduction}

\subsection{Smectic freely suspended films}

Smectic liquid crystals are layered phases of rod-shaped organic molecules, in which each layer is a two-dimensional fluid on the order of a molecular length in thickness. Because of their layering, smectics can readily be made to form films that are freely suspended in air or vacuum. These fluid structures are quantized in thickness, everywhere corresponding to some integral number $N$ of smectic layers, which can be as small as a single molecular monolayer $(\approx 3 \mathrm{~nm}$ thick) for some materials.

Smectic films are structures of fundamental interest in condensed matter physics. They are the thinnest known stable structures of any fluid phase preparation and have the largest surface-to-volume ratio, making them ideal for studies of fluctuations, interface phenomena, and the effects of reduced dimensionality in soft matter. The layering makes films of uniform layer number homogeneous in basic physical properties such as thickness, density, surface tension, and viscosity, to a degree comparable to that of bulk 3D fluids. This distinguishes them from soap films, for example, which generally vary in thickness. Freely suspended smectic films are, except at their edges, completely free of local pinning or other external spatial inhomogeneities. The typically low liquid crystal vapor pressure and the absence of solvent enables the potential for convenient long-term study of such films. The interactions which are operative in ultra-thin films are generally weak, leading to their easy manipulation by external agents such as boundaries, applied fields, and flows, as well as enabling significant fluctuation phenomena with extended spatial correlations. These fluctuation, field, and surface effects, combined with the wide variety of liquid crystal order parameters and symmetries, make freely suspended LC films a rich system for probing basic fluid physics.

\subsection{Fundamental research on thin smectic films}

The initial applications of ultra-thin freely suspended liquid crystals were studies of the structural and fluctuation behavior of smectic $\mathrm{C}$ phases, mesophases in which local molecular tilt from the layer normal creates a $2 \mathrm{D}$ vector field of molecular orientation in each layer plane. This field can be probed optically even in few layer thick films, enabling the direct observation of orientational spin waves [1], quasi-long range ordering [2], compression induced phase transitions [3, 4, topological defects [5, 6] and defect Brownian motion [7, all in 2D. Smectics enabled the first electron microscopy of fluid films [8], and x-ray scattering studies of in-plane molecular ordering in smectic films have provided the most convincing evidence to date for the hexatic phase and its melting behavior 9], a scenario emerging from the theoretical work by Kosterlitz and Thouless [10] for which they won the 2016 Nobel prize in physics. Stable, thin smectic films can be prepared not only in air, but also in aqueous environment [11, 12. Many of these smectic film research themes have been reviewed by Pieranski et al. [13, and Oswald and Pieranski [14]. The OASIS project focuses on smectic 
films as a broad context for the study of a variety of fluid dynamic phenomena in reduced dimensionality, including $2 \mathrm{D}$ hydrodynamics, interfacial-gradient and applied-field driven transport in 2D, and the structure and dynamics of $1 \mathrm{D}$ interfaces in 2D systems. For example, there are significant energy barriers to locally changing the film thickness, since this requires the formation of dislocations associated with the creation or removal of smectic layers. Thus, any flow in a uniformly thin smectic film is essentially confined to the layer plane [14, 15] and conserves the film thickness and density. The continuity equation for the flow field can consequently be reduced to 2D. As a result, a smectic film in a vacuum provides a nearly ideal physical realization of a $2 \mathrm{D}$ incompressible Newtonian fluid [16], quite distinct from soap films which exhibit variable thicknesses 17. If, as is often the case, a smectic film is surrounded by air or some other fluid, motion in the film plane couples strongly to the environment. Due to the momentum exchange between the film and the embedding fluid, the flow in the film displays features of both $3 \mathrm{D}$ and 2D hydrodynamics, behavior that has been dubbed "quasi-2D" hydrodynamics [18. Thus, a smectic film surrounded by a $3 \mathrm{D}$ viscous medium, and containing inclusions such as islands (regions of larger layer number on a few layer thick background film) or particles, is a direct physical analog of protein-containing lipid bilayer membranes.

The hydrodynamics of inclusions in such thin visco-elastic membranes represents a fundamental physical problem and has been the subject of many experiments in different biological, chemical and physical systems, including smectic films in the context of OASIS [19, 20, 21, 22, 23, 24, 25. More broadly, investigations of the motion of inclusions in smectic films offer the opportunity to explore hydrodynamics in restricted geometries [23, 24, 25, 16, 26. Flow under the influence of external electric and magnetic fields [27, 28, 29, 30, 31, 32, 33, mechanical torques [34, and thermal gradients [35, 36] have also been studied. Thin smectic films have also enabled the investigation of hydrodynamic instabilities [37, 38, 39, particle aggregation [40, 41, 42] and quasi-2D emulsions 42, 43, 44, 45, 46, 47, 48.

\subsection{Microgravity experiments}

The vast majority of experiments on freely suspended smectic films have historically been performed on films with planar geometry. Gravity-driven motion can be actively exploited in tilted films to perform microrheology in 2D 24, but under isothermal conditions, gravitational effects on horizontal, flat films can be neglected. If the film geometry is non-planar, however, any inclusions in the film, particularly in the fluid smectic A and C phases, will be susceptible to sedimentation under the influence of gravitation. In order to study the dynamics of such inclusions in non-planar films such as bubbles, one needs to eliminate gravitational effects. Another reason to avoid gravity is to simplify the study of films under nonisothermal conditions. In the presence of gravity, temperature gradients will necessarily lead to buoyancy-driven air convection. Since smectic films are so thin, air flow will readily advect the film material [36], masking intrinsic thermomechanical effects in the films. Simple evacuation of the film chamber does not solve this problem, because in that case the film establishes 
thermal equilibrium with the environment by radiation losses, irrespective of the local thermal boundary conditions imposed by the experimenter 35.

As we shall see below, the influence of the meniscus at the film boundaries on the dynamics of inclusions is greatly reduced by creating spherical films supported only by a thin capillary. Since sedimentation is always an issue in terrestrial experiments performed in this geometry, this motivates the need for investigating smectic bubbles under microgravity conditions.

Microgravity $(\mu \mathrm{g})$ can be realized in several ways, the simplest being drop experiments in a terrestrial laboratory. Drop towers, parabolic flights, sounding rockets and satellites, and earth-orbiting platforms such as the International Space Station (ISS) offer opportunities for more extended access to reduced gravity conditions, on differing time scales and with differing degrees of weightlessness. The use of any of these platforms is contingent on many specific restrictions. For example, spatial constraints, strong initial accelerations, the duration of the reduced gravity phase, and the level of residual accelerations (g-jitter) all affect the feasibility and design of individual experiments. In the following, we will describe the realization of $2 \mathrm{D}$ fluids using smectic liquid crystals to perform hydrodynamic experiments in lg and discuss the technical requirements and design of these experiments. In particular, we are interested in the longterm behavior of inclusions in quasi-2D fluids. The formation, dynamics and coarsening of inclusions in the form of either islands of excess smectic layers or isotropic droplets of the mesogenic material embedded in the liquid crystal film have been studied. We have also observed the response of smectic bubbles to applied electric fields, thermal gradients, and mechanical stresses.

\section{Motivation, materials, and microgravity experiments}

Free-standing films a few nanometers thick in the smectic A (SmA) and smectic $\mathrm{C}(\mathrm{SmC})$ phases are ideal objects for studying hydrodynamic flow patterns and the behavior of inclusions. In these fluid LC phases, the flow is essentially restricted to the plane of the film. In the SmA phase, the preferential direction of the long axes of the rod-like molecules (the director, $\mathbf{n}$ ) is along the smectic layer normal, while in the SmC phase, $\mathbf{n}$ is tilted from the layer normal by an angle $\theta$. Thin SmA films thus are analogues of $2 \mathrm{D}$ isotropic fluids, where all directions in the film plane are equivalent, while thin $\mathrm{SmC}$ films represent anisotropic 2D fluids with polar nematic order. Free-standing films of rod-shaped mesogens can readily be prepared with thicknesses from many micrometers down to two molecular layers $(\sim 5 \mathrm{~nm})$. The overall shape of the films depends upon the geometry of the lateral support and on the pressure difference (Laplace pressure) between the two film surfaces, and smectic membranes have been studied in planar, cylindrical, annular, and spherical geometries. Freely floating smectic films (not attached to any support) have been used to study the shape-dynamics of closed membranes [49, 50] but so far only short-term observations $(t \lesssim 1 \mathrm{~s})$ of such structures have been made.

In most experiments, freely suspended films are bound by a meniscus connecting the film with the support. The shape and extent of the meniscus depend, 
in general, on the geometry, the material, and the conditions under which the film was drawn, but in most cases the meniscus represents a substantial reservoir of material, with a volume far greater than that of the film itself. The meniscus can exchange liquid crystal material with the film in order to compensate, for example, for film thickness changes, growth or shrinkage of islands, changes of the film area, and droplet formation in the films. This exchange is a wellestablished phenomenon [51] that has considerable impact on the film dynamics. The thickness of the roughly wedge-shaped meniscus typically reaches dozens of micrometers, so its structure is truly three-dimensional. The meniscus complicates the execution and interpretation of many hydrodynamic experiments. For example, thermally generated convection in smectic films in vacuum was shown to be driven exclusively by thermal gradients in the meniscus 35, masking intrinsic thermocapillary effects in the films. The meniscus influences the motion and evolution of inclusions in the thin films, affecting the structure and evolution of islands on the film by exchanging material with them and thus interfering with Ostwald ripening of 2D emulsions formed by islands. There have been many attempts at reducing the effects of the meniscus. One approach is to minimize the length of the film boundaries relative to their diameter by inflating the films to form spherical bubbles [52]. In this case, a meniscus forms only at the opening of the supporting capillary, which can be made quite small (with a circumference on the order of a few millimeters), while the bubble diameter can be in the centimeter range. Since on Earth gravity pulls any objects on a film with non-planar geometry downwards, only microgravity conditions allow the long-term study of the evolution or motion of particles, liquid droplets and islands on smectic bubbles. In general, microgravitation offers two important advantages for performing hydrodynamic experiments: (1) There is no sedimentation of material, enabling long-term observations of the motion of inclusions on such curved surfaces. (2) Experiments using thermal gradients are not complicated by the effects of (air) buoyancy, allowing one to observe intrinsic thermocapillary effects.

The aim of the OASIS project (Observation and Analysis of Smectic Islands in Space) was designing, preparing, and conducting experiments on smectic bubbles in the International Space Station (ISS). OASIS represents the first, and so far only, set of experiments devoted to the study of thermotropic smectic liquid crystals in space. In the following, we will introduce the physical questions relevant to thin liquid crystal film dynamics that are addressed by microgravity experiments. We will discuss the specific requirements and technical conditions for carrying out quasi-2D hydrodynamic experiments on smectic bubbles and planar freely suspended films on different microgravity platforms, and summarize the parabolic flight and sounding rocket experiments that were conducted in preparation for the ISS flight.

The main focus of this paper is on describing the OASIS setup and some of the technical issues encountered in the design and execution of the experiments, and showing selected preliminary scientific results of the long-term studies of island and droplet dynamics carried out on the ISS between June 2015 and March 2016. A detailed, quantitative analysis of the flight data will be published 
elsewhere.

\section{Realization of microgravity conditions for experiments on smectic films}

Experiments on smectic films performed under microgravity can be grouped into three categories: The first category includes short-time studies of dynamic processes occurring in the sub-second to second range, such as shape transformations of closed, freely floating smectic membranes. These phenomena were investigated on parabolic flights, which offer up to 22 seconds of $\mu \mathrm{g}$. The second category of experiments includes studies of thermocapillary effects that can be carried out during the few minutes of a sub-orbital rocket flight. The TEXUS rocket experiments facilitated by the DLR (Deutsches Zentrum für Luft- und Raumfahrt) provided a flight opportunity with six minutes of microgravity that is described below. The third category includes long-term observations of inclusion dynamics and the coarsening of two-dimensional island emulsions. For such experiments, time scales of minutes to hours are needed and the only practical way of carrying out these investigations is on the International Space Station.

Each of these experimental platforms provides different conditions and benchmarks. The main experimentally relevant distinctions are the duration and quality of the microgravity phase, the constraints on the technical implementation, repeatability, and the opportunity for direct interaction with the experiment during the $\mu \mathrm{g}$ phase. Of course, another criterion is the expenditure of time and resources, with the ISS experiments requiring a far greater investment of both of these than either the TEXUS mission or the parabolic flights.

A $\mu \mathrm{g}$ environment is also available in the drop tower at ZARM (Bremen), which provides 9.3 seconds of microgravity of excellent quality. We did not consider this as an option for our experiments for several reasons. The acceleration during the initial catapult shot would definitely destroy a thin smectic film. Performing a simple drop experiment instead would circumvent this problem but this would reduce the microgravity phase to less than 4.6 seconds, with at least two hours of preparation required for each drop. The only experiments where such short microgravity periods could be useful are on the shape dynamics of closed bubbles but for these experiments the principal advantage of the drop tower, the excellent microgravity quality, is of little relevance. Parabolic flights, on the other hand, which offer the opportunity of performing 30 experiments per flight with direct interaction of the experimenter, were found to be an efficient and productive way of testing equipment and techniques to be used on the ISS.

\subsection{Parabolic Flights}

Parabolic flights were performed on an Airbus A300 at Novespace in Bordeaux, France. These flights provided the opportunity to perform many repetitions of the experiment (30 per flight) while allowing the experimenter to continuously observe and control the system. 
The 22 seconds of $\mu \mathrm{g}$ during each parabola were sufficient to prepare freely floating bubbles from collapsing catenoid films [53, 49], and to observe the bubble shape dynamics or bubble rupture [49, 50]. The quality of $\mu \mathrm{g}$ achieved during these parabolic flights is not sufficient to perform meaningful hydrodynamic experiments with films attached to a solid support, since the $g$-jitter (Fig. 1. top) of the airplane, which is of the order of several percent of $g$, directly affects the films. Freely floating objects, however, are not as susceptible to such disturbances.

The parabolic flights, undertaken primarily during the 20th parabolic flight campaign of the DLR, were used to test elements of the experimental apparatus to be used in the ISS mission. In addition, we refined the techniques used to prepare free floating smectic bubbles. Short-term observations of smectic islands on the bubble surface and of mechanical instabilities (bubble rupture) were completed mostly during the 27 th and the 29th DLR parabolic flight missions 49, 50.

An example of a freely floating $\mathrm{SmC}$ bubble, which was prepared using the collapsing catenoid technique [53, is shown in Fig. 1. The mesogenic material is a $50 \%: 50 \%$ by weight mixture of 2-(4-n-hexyloxyphenyl)-5-n-octylpyrimidine and 5-n-decyl-2-(4-n-octyloxy- phenyl)pyrimidine, which is in the SmC phase at room temperature. A Rayleigh-Plateau instability of the initially tube-like closed membrane leads to constriction about its diameter and eventually to the formation of individual, spherical bubbles. As a consequence of the reduction of the bubble surface area by capillary forces, the excess smectic material forms islands. When viewed in parallel, transmitted light, these islands appear as dark spots on the films (see Fig. 1). The long-term study of the dynamics of such smectic islands was one of the main goals of OASIS.

\subsection{Sounding rocket experiments}

With sounding rockets, experimental microgravity times of several minutes' duration can be achieved. This is sufficient to analyze, for example, thermally driven convection in thin, planar liquid crystal films. As intimated above, smectic films would not withstand the huge acceleration experienced during the launch phase and are therefor only prepared during the flight. The weightless phase is not long enough to create smectic bubbles, which require several minutes of careful inflation, but planar films can be drawn within less than a minute, leaving enough time to perform thermocapillary experiments. In preparation for the OASIS mission, the OASIS-TEx (Thermocapillary EXperiment) was successfully launched with TEXUS 52 in Esrange, Sweden on April 27, 2015. The rocket reached an altitude of about $250 \mathrm{~km}$ and provided approximately $360 \mathrm{~s}$ of weightlessness of much better quality than available on the parabolic flights. The TEXUS mission, however, offered only limited opportunities for providing feedback and controlling the experimental parameters during the flight. These experiments provided the first observations of thermocapillary drift and convection in smectic films exposed to in-plane temperature gradients in $\mu \mathrm{g}$ [54. The setup for the thermocapillary experiment is shown in Fig. 2 . LEDs were used for illumination and the film was observed in reflection using 

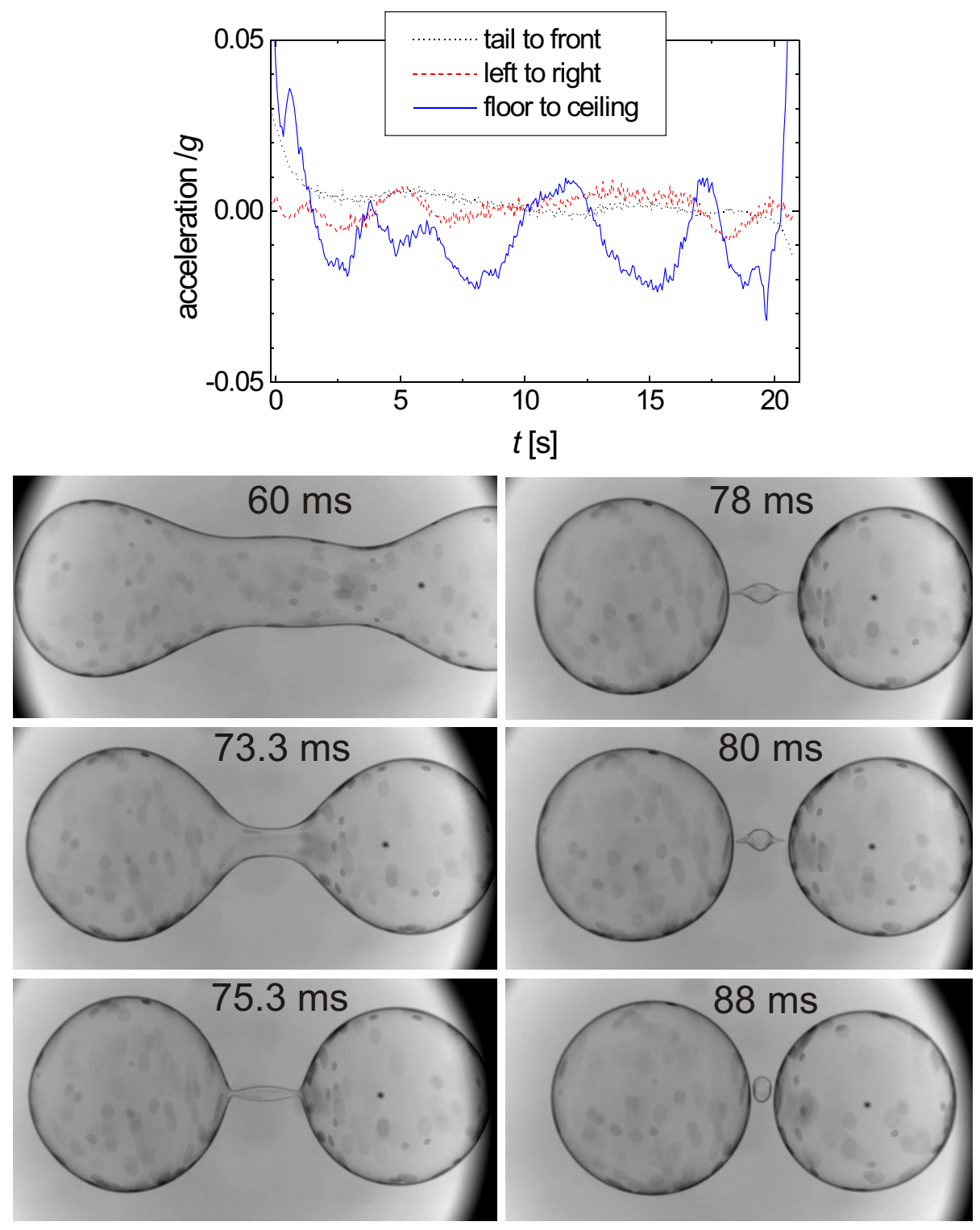

Figure 1: Top: Typical acceleration profiles during a parabolic flight (parabola 00 of Novespace flight 548, on February 11, 2014). The vertical acceleration trace (blue) shows the relatively large deviations from weightlessness that are most problematic for our experiments. The forward and left/right accelerations (green and red curves, respectively) are less critical. Bottom: Evolution of a freely floating smectic bubble. The time is given relative to the initial pinch-off of the bubble from a collapsing catenoid. A Rayleigh-Plateau instability causes the initially elongated bubble to collapse, becoming dumbbell-shaped before pinching off in the middle, leading to the formation of two large bubbles and a smaller daughter. The image dimensions are $22 \mathrm{~mm} \times 11 \mathrm{~mm}$. The dark spots are islands of excess smectic material floating on the film. 
a CCD camera. Two heating/cooling blocks in contact with the film were used to generate thermal gradients and changes in the SmC Schlieren texture were used to track flow in the film plane. The material used in these experiments was 5-n-octyl-2-(4-n-octyloxyphenyl)pyrimidine, with the mesophase sequence: Isotropic $68^{\circ} \mathrm{C}$ Nematic $62^{\circ} \mathrm{C} \mathrm{SmA} 55.5^{\circ} \mathrm{C} \mathrm{SmC} 28.5^{\circ} \mathrm{C}$ Cryst.

\subsection{ISS experiments}

For long-term experiments on spherical smectic films (bubbles) supported by a thin needle under microgravity conditions, the OASIS setup was successfully launched at the Kennedy Space Center on a SpaceX Falcon 9 Rocket on April 17, 2015, and was transported to the ISS in the Dragon CRS-6 module. Experiments were performed until March 2016. The following sections describe the essential technical details of OASIS and give some preliminary results.

\section{OASIS setup and technical implementation}

The primary goal of the OASIS experiments on the ISS was to carry out investigations of hydrodynamic flow in smectic freely suspended films, the relaxation of hydrodynamic perturbations, and the long-term behavior of emulsions of islands and droplets. Experiments were also performed to study thermocapillary phenomena and the effects of applied electric fields and mechanical stresses on the bubbles. The microgravity conditions on the ISS prevented sedimentation and eliminated buoyancy-driven thermal convection of the surrounding air and in the liquid crystal films. A bubble diameters of $15 \mathrm{~mm}$ was considered sufficiently large to minimize the influence of the meniscus around the bubbleinflation needle on the dynamics of inclusions in the film. Film thicknesses were expected to be in the range between about 5 and several hundred nm (i.e., from 2 to 100 or more smectic layers), with thin films preferred as the background of island emulsions, and thicker films being more suitable for preparing droplet arrays by melting islands into the nematic or isotropic phase.

We proposed to study emulsions of islands created primarily by shear flow of the films. Islands can be used as tracers to visualize flow on the bubble surface, and their size and shape can be monitored in order to characterize the dynamical behavior of $2 \mathrm{D}$ island emulsions. The mutual interactions of inclusions and their interactions with topological and layering defects are also of interest. We also planned to shoot picoliter-sized droplets of an immiscible isotropic liquid onto the bubbles using a microdispenser and to study their behavior. In addition to mechanical manipulations of the smectic bubbles by controlled inflation, deflation and shear flow in the film plane, the experimental apparatus was designed to allow the study of the influence of thermal gradients and electric fields.

Apart from the long-term character of the experiment, the ISS provides an environment where the quality of microgravity is much better than in parabolic flights. For comparison with the parabolic flight conditions, we show a 20 second sequence of typical acceleration data measured during our experiment in Fig. 3 . 
a)
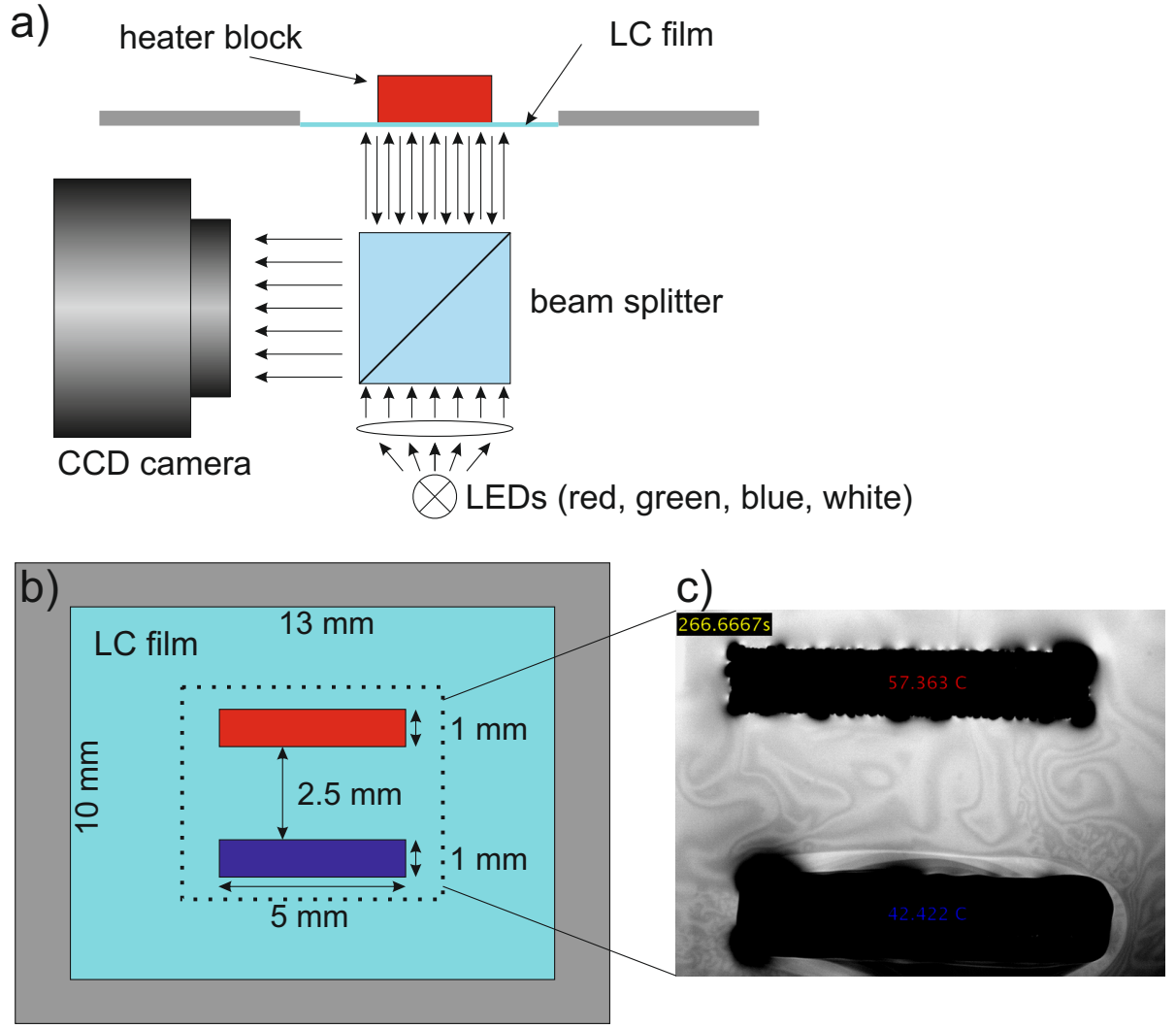

Figure 2: OASIS-TEx setup for thermocapillary experiments on smectic films in microgravity. (a) Side view of the illumination system. The film is observed in reflection using monochromatic or white polarized light provide by LEDs. (b) Film and heater geometry. The two rectangular objects (red/blue) are heating/cooling blocks that contact the film. These can be set to temperatures $T_{0} \pm \Delta T$ above and below ambient to produce temperature gradients of up to $10^{\circ} \mathrm{C} / \mathrm{mm}$ in the film. c) Reflection image of Schlieren textures in a $535 \mathrm{~nm}$ thick $\mathrm{SmC}$ film. Changes in the texture help to visualize thermally-induced vortex flow, which sets in at the highest temperature gradients achieved in the experiment. The film chamber is at $T_{0}=50^{\circ} \mathrm{C}$. Material parameters and details are given in Ref. [54].(For interpretation of the references to color in this figure legend, the reader is referred to the web version of this article.) 


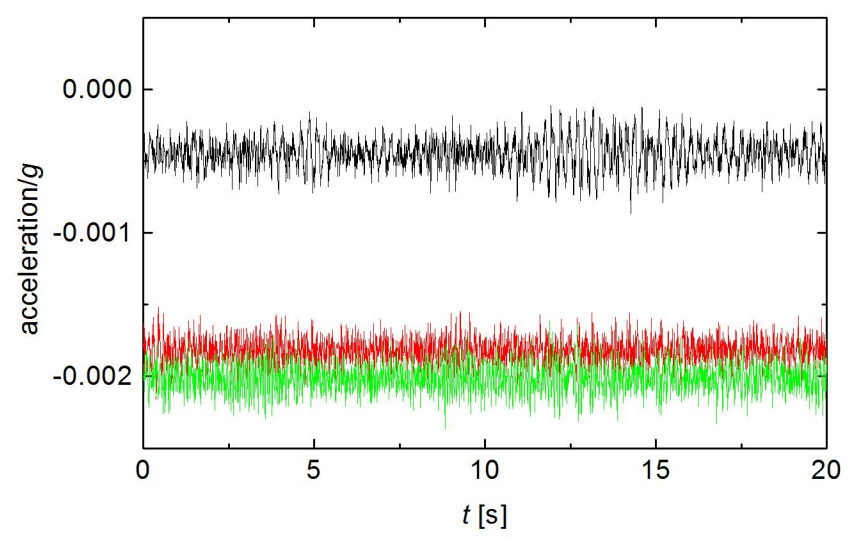

Figure 3: Typical example of acceleration data from the MSG, measured at the OASIS Floor Plate on Nov. 16, 2015, starting at 14:35:23.503. Black, red and green colors (top to bottom curves) refer to the three acceleration components $a_{x}, a_{y}, a_{z}$ in the coordinate system of the sensor. High frequency oscillations $(>50 \mathrm{~Hz})$ are practically irrelevant for our experiments. The overall quality of $\mu \mathrm{g}$ is substantially better than in parabolic flights (c.f. Fig. 1. top).

The OASIS hardware was installed in the Microgravity Science Glovebox (MSG) on the ISS. A sketch of the MSG with the OASIS hardware installed is shown in Fig. 4, left. The main component is the bubble chamber enclosure that contains the experimental setup, including illumination and observation elements, shown at right. The individual elements of the bubble chamber enclosure are described in detail below.

\subsection{Liquid crystal materials}

Four different liquid crystal samples (SN001-SN004) were selected for the ISS flight. A decisive criterion for selection was that the materials have fluid smectic phases at room temperature and that they could readily be blown into bubbles. We also wished to compare the behavior of chiral and non-chiral, polar and non-polar, tilted and non-tilted phases.

- SN001: Polar SmA (mixture of 8CB + Displaytech MX 12160), Isotropic $56^{\circ} \mathrm{C}$ Nematic $54^{\circ} \mathrm{C} \mathrm{SmA} 5^{\circ} \mathrm{C}$ Cryst.

- SN002: Racemic SmC (Displaytech MX 12846), Isotropic $84.7-82.0^{\circ} \mathrm{C}$ Nematic $81.4^{\circ} \mathrm{C} \mathrm{SmA} 66.1^{\circ} \mathrm{C} \mathrm{SmC}$

- SN003: Chiral SmC* (Displaytech MX 12805), Isotropic $84.7-82.0^{\circ} \mathrm{C}$ Cholesteric $81.4^{\circ} \mathrm{C} \mathrm{SmA}^{*} 66.1^{\circ} \mathrm{C} \mathrm{SmC} *$

- SN004: Non-Polar SmA (Displaytech MX 12160), Isotropic $51.1^{\circ} \mathrm{C} \mathrm{SmA}-3.2^{\circ} \mathrm{C}$ Cryst $\left(3.1^{\circ} \mathrm{SmA}\right)$. 

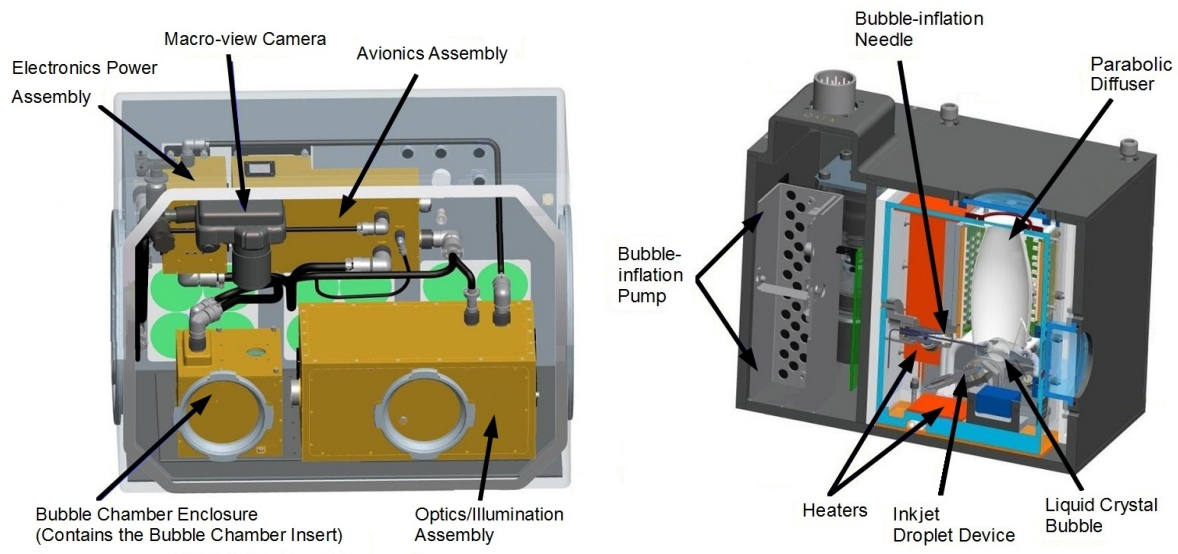

Figure 4: The OASIS experiment was housed in the Microgravity Science Glovebox, located in the Destiny Module of the ISS. Left: Overview of the OASIS hardware. Right: Detailed view of the Bubble Chamber enclosure.

$8 \mathrm{CB}$ is $4^{\prime}$-n-octyl-4'-cyanobiphenyl, obtained from Sigma-Aldrich. The Displaytech materials are proprietary mixtures supplied by the Miyota Development Center of America.

A separate bubble chamber enclosure and dispenser system was used for each sample. The samples were exchanged periodically by ISS crew members but the experiments were controlled from NASA Glenn Research Center's ISS Payload Operations Center in Cleveland, Ohio. The total amount of each LC sample available for the experiments was about $100 \mu \mathrm{l}$.

\subsection{Bubble-inflation needle}

The primary task in each experiment, and the basis of all subsequent measurements, was smectic bubble generation using a concentric needle device (Fig 5). At the beginning of each experiment, a small amount of liquid crystal material was driven through the outer needle until a thin cap formed across the opening of the inner needle. This was done carefully, avoiding excess material that might wet the outer needle surface. If this happened, the bubble would slide down the needle or become canted in the bubble chamber, in which case the capillary would have to be cleaned by heating the setup far into the isotropic phase of the mesogen. Once a cap had formed, air was pumped through the inner needle to generate a thin, oriented film which was then gently inflated to become a spherical bubble. Bubble inflation was carried out at high temperatures in the smectic phase, where the viscosity of the material is low, achieved by heating the needle assembly using a Nichrome wire heater wrapped around it. During inflation, the needle temperature was set in the range from $25^{\circ} \mathrm{C}-$ $75^{\circ} \mathrm{C}$, depending on the liquid crystal material. The bubbles were inflated using a Cavro Xcalibur $30 \mathrm{~mm}$ Stroke Syringe Pump. The maximum pump rate was $0.83 \mathrm{ml} / \mathrm{sec}$, although in order to inflate thin, stable bubbles, the flow-rate could 
be reduced to $0.21 \mu \mathrm{l} / \mathrm{sec}$. In general, the inflation speed determined the film thickness, with faster inflation leading to thinning of the films. Rapid deflation of the bubbles (for example, in order to create islands) was limited by the pumping rate. With bubble volumes of almost $2 \mathrm{ml}$, the fastest possible deflation took a few seconds.

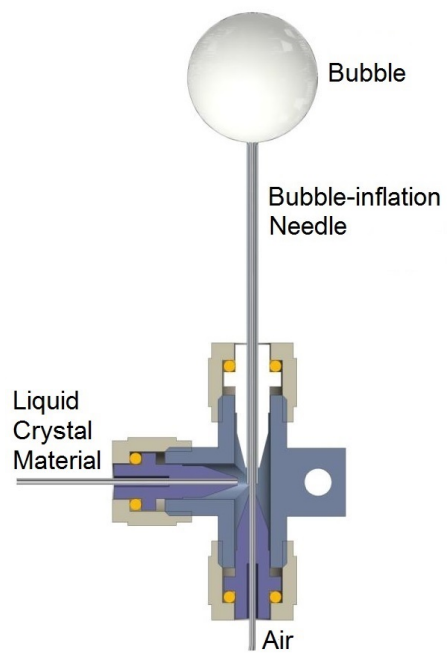

Figure 5: Concentric needle device used to generate and inflate liquid crystal bubbles. The inner capillary (outer diameter $0.72 \mathrm{~mm}$, inner diameter $0.41 \mathrm{~mm}$ ) is connected to an air pump for inflation and deflation of bubbles. The gap between the outer capillary (outer diameter $1.27 \mathrm{~mm}$, inner diameter $0.84 \mathrm{~mm}$ ) and the inner capillary is filled with liquid crystal material pumped from a larger reservoir that is used to form an initial thin film over the opening of the inner needle.

\subsection{Macro-view and micro-view cameras}

All visual observations of the bubbles were made using two identical, highresolution, color CCD cameras (Prosilica GX 1050C, Allied Vision Technologies). An external optics/illumination assembly (shown in Fig. 6) housed the micro-view camera and a spectrometer used for film thickness measurements. The micro-view camera was mounted on a simple reflected light microscope equipped with a $10 \times$ objective (10x Mitutoyo Plan Apo SL Infinity Corrected Objective, Edmund Optics PN NT46-144) used to obtain detailed images of film textures. The objective, which has a $15 \mathrm{~mm}$ working distance, was positioned to image the top of the bubble located between the airjets, with a field of view of $500 \times 500 \mu \mathrm{m}^{2}$ and a depth of field of $3.5 \mu \mathrm{m}$. The camera could be translated laterally using two linear positioners. The cameras support a maximum frame rate of $112 \mathrm{fps}$ but in the OASIS experiments, a maximum rate of $60 \mathrm{fps}$ was used. The micro-view camera allowed us to observe such phenomena as the coalescence and coarsening of islands, and hydrodynamic effects caused by applied electric fields, thermal gradients and the airjets, with high resolution. The 
numerical aperture of the micro-view camera was 0.28 and the spatial resolution about $0.5 \mu \mathrm{m}(1024 \times 1024$ pixels $)$. The spectral sensitivity range of the camera was from $350 \mathrm{~nm}$ to $1000 \mathrm{~nm}$.

The system could be converted to a polarizing microscope by inserting linear glass polarizing filters (Edmund Optics PN TN43-785) with an efficiency of $95 \%$. The LED illuminator (DiCon G180 Series ScopeLED) had a color temperature range of $2700 \mathrm{~K}$ to $7500 \mathrm{~K}$ (we selected $6500 \mathrm{~K}$ ) and a maximum output of $975 \mathrm{~lm}$. For interferometric measurements of the thickness of the films and islands, a spectrometer (StellarNet BLUE-Wave PN UVN) with a wavelength sensitivity in the range $250 \mathrm{~nm}$ to $1100 \mathrm{~nm}$ was available. The spectral resolution was $6 \mathrm{~nm}$ with the option to improve it to $1 \mathrm{~nm}$.

In addition, each bubble chamber enclosure was equipped with a macroview camera (see Fig. 4), which imaged the entire bubble. Achieving uniform illumination of the bubble was one of the biggest challenges in designing the OASIS hardware. The bubble was positioned inside a translucent, parabolic diffuser that was illuminated from the outside by several arrays of LEDs. The macro-view camera allowed us to observe the bubble during the inflation stage (and monitor its diameter afterwards), and was used to record large-scale phenomena such as thermocapillary flow, coarsening dynamics (including Ostwald ripening and the coalescence of smectic islands and thinner, circular domains in the film called "holes"), and hydrodynamic instabilities in 2D. The macro-view camera was equipped with a $50 \mathrm{~mm} \mathrm{f} / 2.8$ macro lens (Sigma EX DG Macro, PN 346101 ) and provided an image with much lower magnification than the microview camera, the resolution being about $18 \mu \mathrm{m}$ per pixel. During the flight, a data downlink provided low-resolution images from both cameras, examples of which are shown in Fig. 7

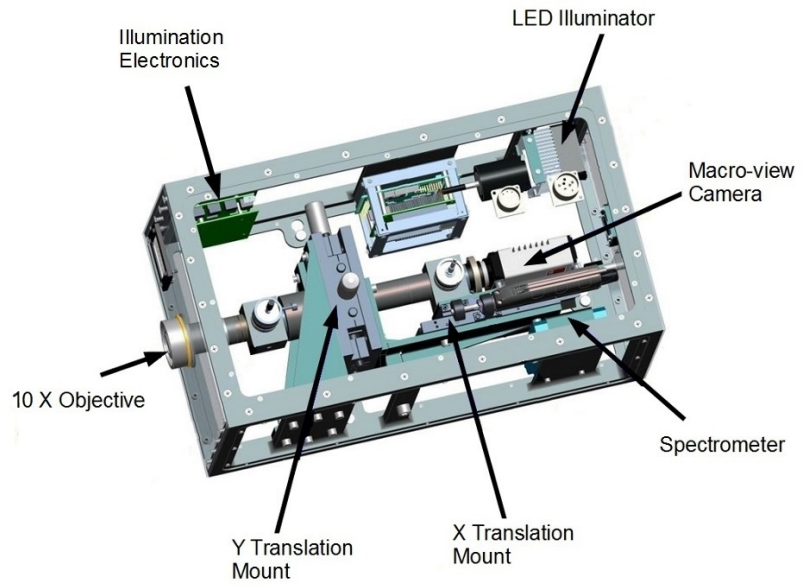

Figure 6: Optics/illumination assembly with micro-view camera, spectrometer, objective and LED illuminator. 


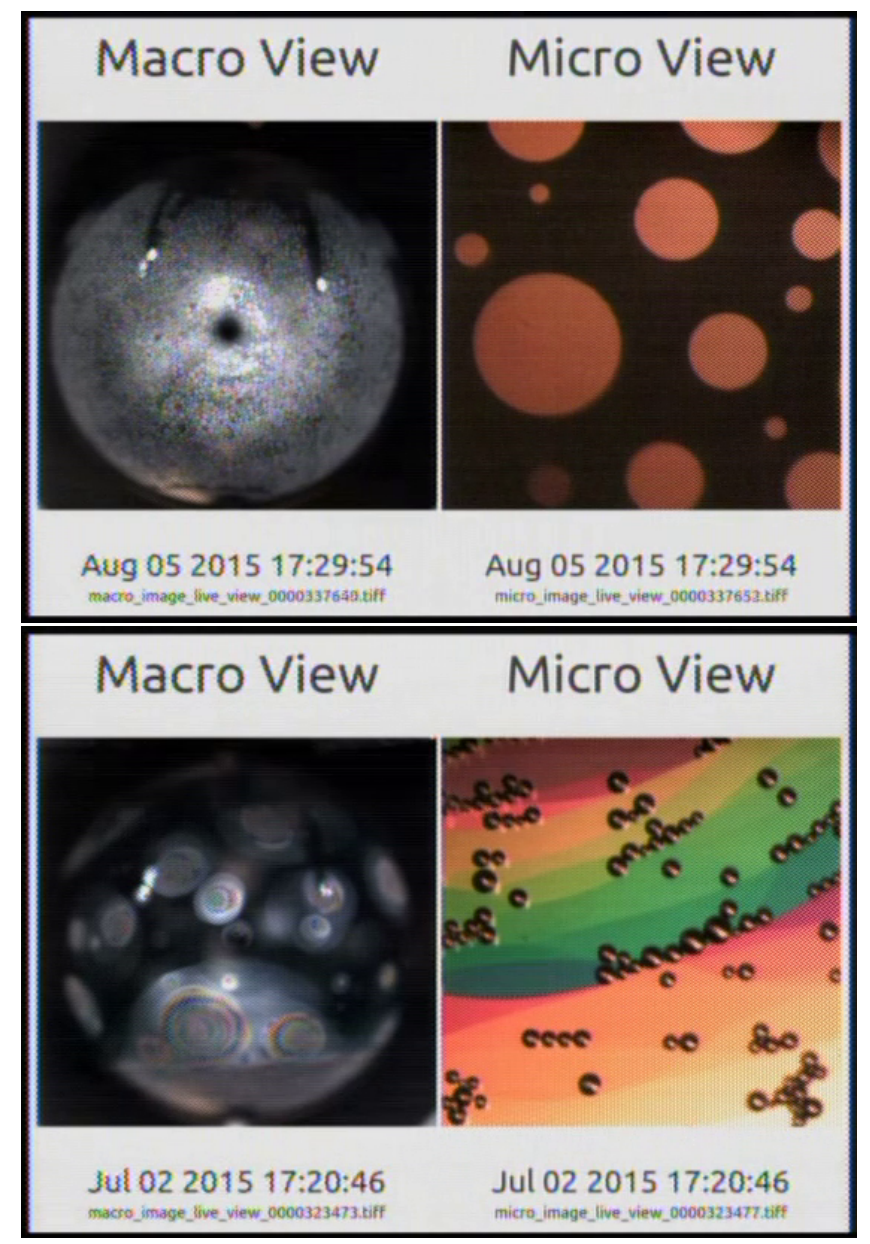

Figure 7: Typical macro- and micro-views of the smectic bubble experiment captured from the live downlink. The macro-view images show the entire bubble. The bubble-inflation needle is just out of frame at the bottom of these images and curved reflections of the airjet needles can be seen near the top of the upper bubble. The corresponding micro-view images show smectic islands on a thin background film (top) and molten droplets pinned to layer steps in a thick background film (bottom). The different interference colors are indicative of the film thickness. The resolution of the downlinked images is $512 \times 512$ pixels. 


\subsection{Airjet needles}

Several techniques could be used to create islands on uniformly thick smectic films. The most convenient was the application of strong extensional air flow along the film surface using four air jets, shown in Fig. 8, Filtered station air (supplied by the MSG) blown along the bubble surface led to continuous flow of material all around the bubble. As a result of this flow, excess material in the meniscus could be drawn onto an initially uniform bubble and then broken up into an emulsion of small islands. Alternatively, existing arrangements of islands and holes could be redistributed or randomized in order to reinitialize the system and start a new experiment. Hydrodynamic instabilities could also be triggered. The airjet needles provided controllable air flow of between 10 and $150 \mathrm{sccm}$ (standard cubic $\mathrm{cm}$ per minute). In addition, an electric field could be applied between two of the opposing needles, whose tips were about $2 \mathrm{~mm}$ apart. A third needle could be heated to generate temperature gradients on the bubble surface (further details are given below).

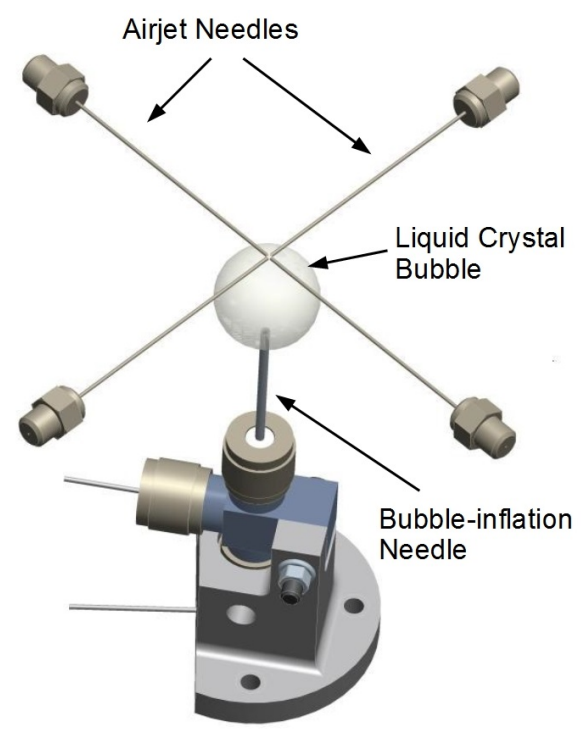

Figure 8: Airjet and bubble-inflation needles. Four independent airjets oriented tangentially to the top of the bubble allowed the creation of flow in arbitrary directions along the bubble surface. One of the needles could be heated to perform experiments under non-isothermal conditions. Two of the needles also served as electrodes to apply in-plane AC or DC electric fields to the smectic film. The needles were fixed in the chamber the distance between the airjets and the smectic film depending on the bubble diameter.

\subsection{Temperature control}

The bubble chamber could be heated uniformly above station temperature (which varied between 20 and $25^{\circ} \mathrm{C}$ ) up to $60^{\circ} \mathrm{C} \pm 0.5^{\circ} \mathrm{C}$. This was achieved 
using Kapton (polyimide) encapsulated electrical heaters (Omega KHLV-103 Series) distributed on the aluminum bubble chamber walls.

Temperature control allowed the study of the temperature dependence of dynamic parameters under isothermal conditions. We were also able to heat bubbles to the smectic-nematic or smectic-isotropic transition, where layer-bylayer thinning of the films (melting of the inner layers and redistribution of the molten material) leads to the creation of nematic or isotropic droplets on the film. The self-organization of those droplets, their dynamics, and their evolution as a function of temperature, were some of the interesting phenomena that could be explored under microgravity conditions.

An important goal of the OASIS project was to investigate the effects of thermal gradients on thin films and any embedded inclusions. The temperature dependence of the surface tension of the LC material and changes in the orientational order parameter were expected to contribute to thermally-induced drift and convection in the films. Buoyancy-driven convection was eliminated in zero gravity. Temperature gradients could be applied by heating either the bubble-inflation needle or one of the airflow needles to higher temperature than the rest of the chamber. For this purpose, one airjet needle was wrapped with Nichrome wire, which allowed us to heat the needle to a maximum of $75^{\circ} \mathrm{C}$. The distance between the heated airjet needle and the bubble surface was determined by the bubble diameter. Temperature differences of about $30^{\circ} \mathrm{C}$ could be realized across the $15 \mathrm{~mm}$ bubble.

\subsection{Electric fields}

Liquid crystal materials couple dielectrically to applied electric fields. In chiral, tilted mesophases there is also ferroelectric coupling to the spontaneous polarization. In $\mathrm{SmC}$ and $\mathrm{SmC}^{*}$ phase films, applied fields cause reorientation of the in-plane tilt direction, an effect that is clearly visible in a reflected polarized light microscope. This reorientation of the director field may lead to the rearrangement of islands or droplets in such films. Any charged inclusions in either SmA or SmC films will experience Coulomb forces in an applied field, and the presence of ions in the liquid crystal material may lead to electrically driven convection, especially in low frequency or DC fields [37, 38, 39]. Two of the airflow needles were therefore configured as electrodes. The tips of the needles were about $2 \mathrm{~mm}$ apart and a maximum voltage of $100 \mathrm{~V}$ (DC) could be applied between them. The airjets were typically located $\sim 1 \mathrm{~mm}$ above the bubble. The applied voltage was generated using an operational amplifier circuit (included in the avionics package of the MSG). The frequency could be varied from $\mathrm{DC}$ to $10 \mathrm{kHz}$, in steps of $10 \mathrm{~Hz}$ between 0 and $1 \mathrm{kHz}$ and in steps of $100 \mathrm{~Hz}$ between $1 \mathrm{kHz}$ and $10 \mathrm{kHz}$.

\subsection{Droplet dispenser}

In addition to the observation of smectic islands and holes on the bubble surfaces, we proposed to investigate the behavior of isotropic liquid droplets

embedded in the film. The plan was to shoot micrometer-sized glycerol/water 
droplets (from a mixture of water and less than $5 \%$ glycerol) onto the bubble surface using an inkjet droplet dispenser [55. The dispenser (MicroFab Technologies MJ-ATP-01-030-DLC) had a $30 \mu \mathrm{m}$ dispensing diameter and was designed to eject between 1 and 500 droplets per second. This dispenser was positioned near the base of the bubble, and could be rotated azimuthally using a stepper motor. The inkjet device failed to work during the ISS mission, with no droplets generated at all. Instead, we produced droplets by heating the material to the upper limit of the smectic temperature range, as described above. This approach is not straightforward, with the heating protocol (both the heating rate and final temperature) being critical, and the bubbles highly susceptible to rupture near the phase transition. Such thermally induced droplets do not nucleate homogeneously, appearing in random positions in the film, and it was not possible to confine them to certain areas or to distribute them homogeneously on the bubble surface. Their size distribution, however, turned out to be rather uniform. This method of creating droplets by heating could only be carried out with the two SmA samples because they had phase transition temperatures within the nominal operating range of the bubble chamber. With the $\mathrm{SmC}$ materials, the transition temperatures were too high.

\section{Preliminary results}

The scientific output of the TEXUS and parabolic flight missions performed in preparation for the OASIS mission have been outlined above. The majority of the OASIS experiments performed on the ISS were very successful and most of the scientific goals were achieved. The OASIS hardware was installed in the MSG by Cosmonaut Gennady Padalka on June 21, 2015. Sample changeouts, hard drive swaps, and glycerol/water fills were subsequently performed by Cosmonaut Oleg Kononeko and Astronauts Scott Kelly, Tim Peake, and Tim Kopra. Low-resolution downlink video data have been available since the start of the mission in March 2015. Hard drives containing high definition imagery recorded on the ISS have recently been returned to Earth and will be used to analyze the experiments in detail. The following preliminary results are based on an initial, qualitative evaluation of the downlink data.

Coarsening of $2 D$ emulsions of smectic islands. Macro-view images recorded during a 42 minute coarsening experiment of smectic islands are shown in Fig. 9 The average initial island diameter can be controlled by the amplitude and duration of the shear induced by the airjet needles, with the distribution of island sizes immediately after the shear flow is stopped being rather narrow. On the ISS, the Perrin length $L=k_{B} T /(m g)$, a characteristic height associated with sedimentation of particles with mass $m$ under gravity, is much larger than the bubble diameter (on Earth, it is comparable to the island diameter). The islands are initially distributed fairly uniformly on the bubble surface, as seen in the first image of Fig. 9. The line tension associated with the dislocations at the island boundaries causes the islands to be circular. The dark background film is only a few smectic layers thick, while the islands have dozens of layers. 
Over time, the island emulsion coarsens significantly, with the average island diameter increasing and the total number of islands decreasing. This is achieved both by Ostwald ripening (the effective diffusion of LC material from smaller islands through the uniform thin background film to larger islands) and by island coalescence. In the experiment depicted in Fig. 9, a large island eventually becomes trapped at the bubble-inflating needle. An example of the redistribution of island material by Ostwald ripening recorded with the micro-view camera is shown in Fig. 10. Here we see two small islands located between several larger ones shrinking and disappearing over a 21 minute period.
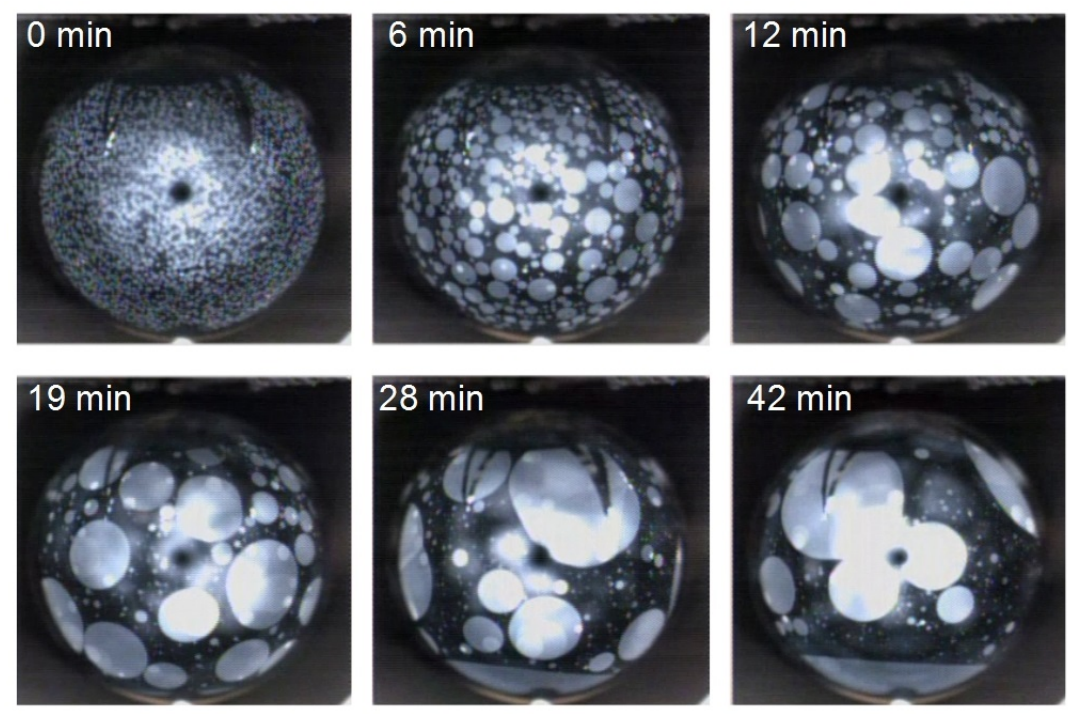

Figure 9: Coarsening dynamics of smectic islands on a thin SmA bubble observed over a period of 42 minutes. At $t=0$, the initial $2 \mathrm{D}$ island emulsion, prepared by shear flow using two opposed airjet needles, is a fairly monodisperse collection of small, disk-shaped smectic inclusions. The emulsion coarsens over time, by Ostwald ripening and coalescence. The bubble is approximately $15 \mathrm{~mm}$ in diameter. The tip of the bubble-inflation needle is visible at the bottom of these images. The material is SN001 and the chamber temperature $35^{\circ} \mathrm{C}$.

Thermally-induced island migration. When the bubble-inflation needle is set to a temperature higher than the environment temperature of the chamber, a slow, thermally-induced migration of objects on the film against the temperature gradient, towards the colder regions, is observed. The islands move with velocities on the order of $10 \mu \mathrm{m} / \mathrm{s}$. When, instead, two airjet needles near the top of the bubble are heated, the direction of migration is reversed, with the islands now moving towards the bottom of the bubble. Typical scenarios of island reorganization in a bubble in such temperature gradients are shown in Fig. 11 .

Self-organization of droplets. Droplets created in SmA films are subject to two self-organizing processes. In inhomogeneous films, the droplets tend to cluster 

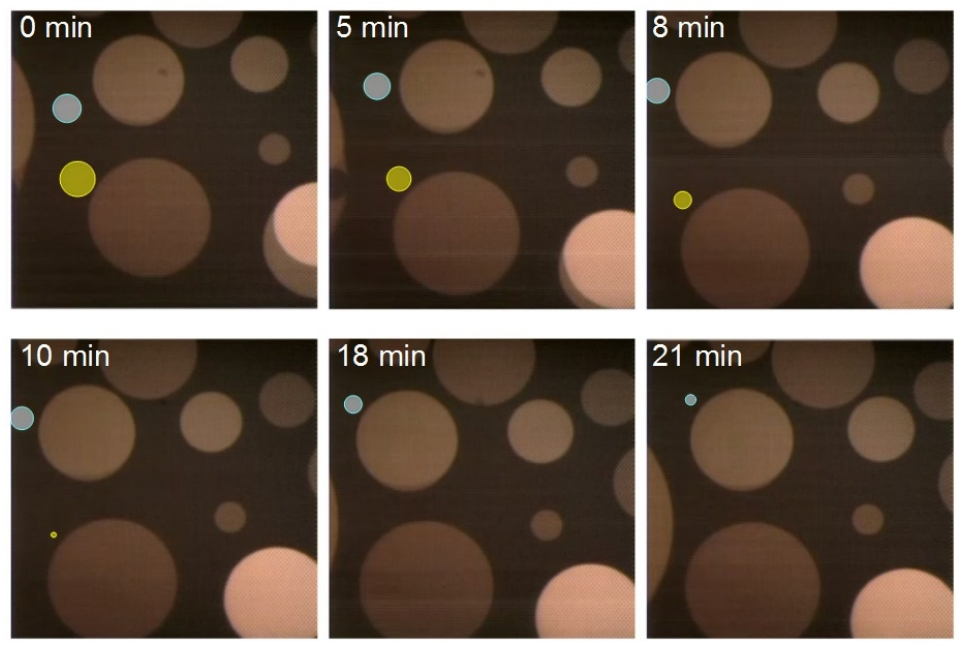

Figure 10: Ostwald ripening of a SmA island emulsion observed with the micro-view camera over a period of 21 minutes. Two small islands (highlighted here in false color) shrink and disappear. The material is SN001 and the chamber temperature $24^{\circ} \mathrm{C}$.

along layer thickness steps, on the thicker plateau but pinned to the dislocation [56, 57, as shown in Fig. 12, left. In SmA films of uniform thickness, molten droplets are seen to self-assemble into remarkably regular, hexagonal lattices, illustrated in Fig. 12 , right. The nature of the mutual interactions between the droplets leading to this latter behavior is not understood at this time.

Further island/droplet experiments were performed by rapidly shrinking then re-inflating the bubbles. Rapid shrinkage leads to a quick reduction of the film area that cannot be compensated by flow into the bubble meniscus. The excess material instead forms islands on the films. One of the aims of the OASIS experiment was to investigate the influence of deflation speed and deflation ratio on the size distributions and densities of the resulting island emulsions. These experiments should provide insight into dislocation dynamics in smectics.

\section{Summary}

\subsection{Achievements}

Parabolic flights provided the opportunity to perform experiments on freely floating, closed smectic bubbles and yielded interesting results on their shape transformations and rupture dynamics [49, 50]. The sub-orbital TEXUS mission allowed us to observe thermocapillary effects in thin free-standing films in air in $\mu \mathrm{g}$. The data from these experiments have been evaluated and the findings are being prepared for publication.

The ISS experiments led to novel insights into the coarsening dynamics of $2 \mathrm{D}$ emulsions of smectic islands on spherical films, which were seen to proceed both 

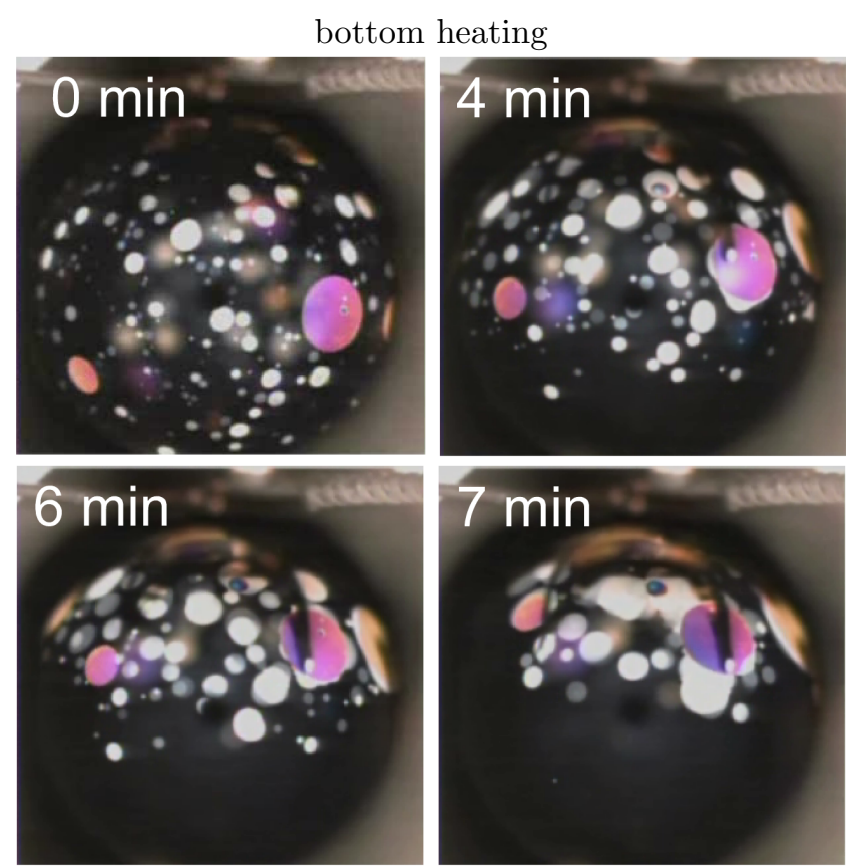

top heating
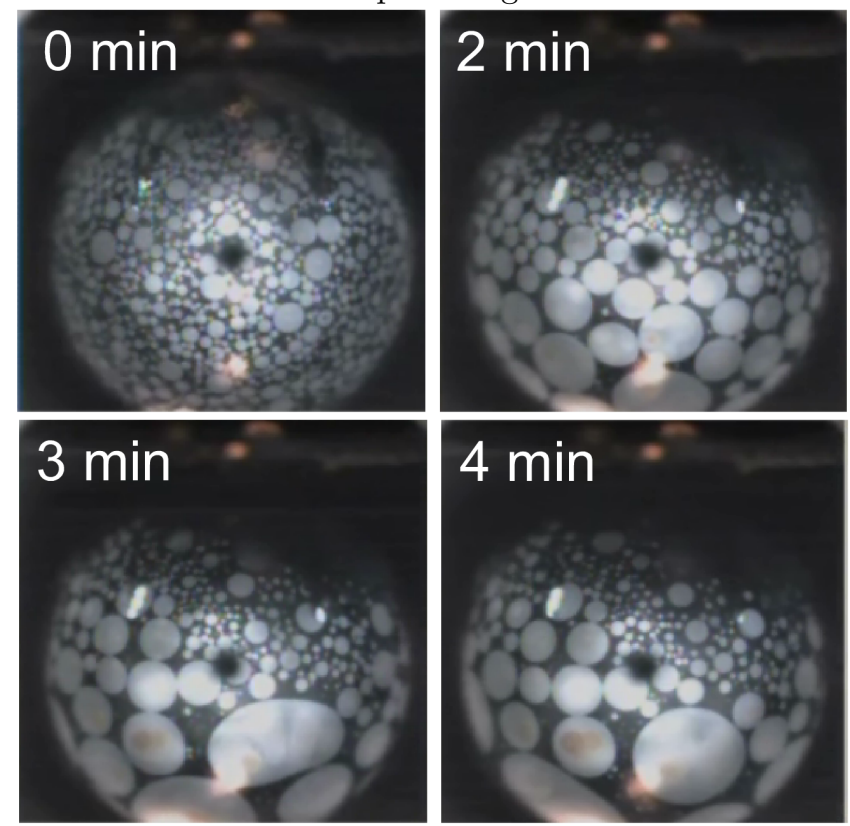

Figure 11: Thermocapillary flow in a SmA bubble caused by heating the bubble-inflation needle, located just out of frame at the bottom of the bubble (top sequence), or by heating two of the airjet needles, located just above the top of the bubble (bottom sequence). In both cases, the islands are transported to the colder side of the bubble, against the temperature gradient. The material was SN004 and the chamber temperature $25^{\circ} \mathrm{C}$. The bubble-inflation and airjet needles were heated in turn to $45^{\circ} \mathrm{C}$. 

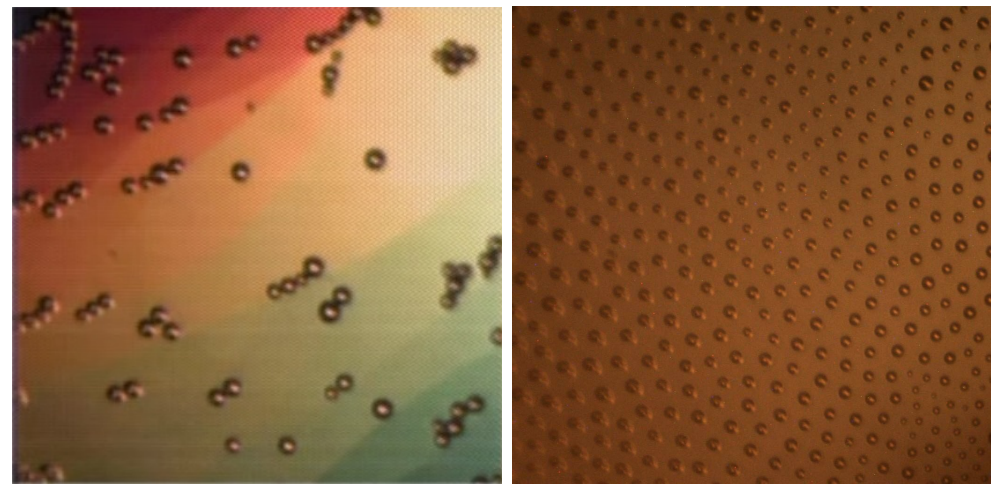

Figure 12: Left: Small nematic microdroplets (with diameters of around $50 \mu \mathrm{m}$ ) assemble and form chains at layer steps in an inhomogenously thick SmA film with terraces. This is a consequence of capillary interactions [56, 57. The material is SN001 and the chamber temperature is $55^{\circ} \mathrm{C}$. Right: In uniformly thick $\mathrm{SmA}$ films without layer steps, molten isotropic microdroplets self-organize in a more or less regular hexagonal lattice. The material is SN004 and the chamber temperature is at the clearing point (smectic-isotropic transition) of the bulk material. In the films, this transition occurs at slightly higher temperatures than in the bulk. These images were recorded with the micro-view camera.

by island coalescence and Ostwald ripening. Island formation and disappearance during bubble deflation and inflation, respectively, were observed and recorded. Migration of inclusions in thermal gradients established across the bubble, and the ordered self-assembly of microdroplets on the curved bubble surface, were observed. Following the successful return of the OASIS hard drives to Earth, detailed evaluation of the data is now in progress.

\subsection{Technical problems}

Several technical problems had to be overcome during the ISS experiments. The biggest issue was the malfunction of the droplet dispenser. All attempts at shooting glycerol/water droplets onto the bubbles failed. It appears that during the mission, the inkjet device did not produce any droplets at all, for reasons that are not clear. While under normal gravity it was found to be quite straightforward to fill the dispenser with bubble-free fluid, microgravity conditions on the ISS may have led to incomplete filling, with air trapped in the dispenser body preventing droplet ejection. A workaround during the mission was the creation of droplets from molten smectic material. This was possible for only two of the flight samples, however, the phase transition temperatures of the other two liquid crystal materials exceeding the designed temperature range of the bubble chamber.

In general, focusing the micro-view camera turned out to be a tedious and time-consuming procedure, particularly whenever a new bubble was inflated or the bubble diameter changed. Once the focal plane was established, however, the micro-view camera worked very well. Towards the end of the mission, the micro-view camera experienced another technical issue, when malfunction of 
the linear positioners prevented the camera from being translated to different locations, which greatly restricted its usefulness.

Overall, these problems demanded some improvisation and flexibility in carrying out the planned experimental procedures but they hardly affected the successful outcome of the OASIS mission.

\subsection{Outlook}

The OASIS experiments have demonstrated the advantages of microgravity in performing fundamental investigations of thin, smectic liquid crystal films. It is already clear, even before any quantitative evaluations have been completed, that these experiments represent only the beginning of the exploration of this field. Many new questions have arisen, related to the basic physics of liquid crystals and the hydrodynamics of thin, fluid films. For example, the experiments suggest ways of exploring the local dynamics of freely floating films, where processes like budding and wrinkling have been observed [49, 50]. Further experiments should help elucidate their dependence on the fundamental physical properties of smectic membranes. Thermocapillary experiments in $\mu \mathrm{g}$ have revealed unexpected behavior in planar films. In future experiments, studies under well-defined thermal conditions are needed to understand quantitatively the fluid migration induced by temperature gradients in quasi-2D smectic films. A TEXUS reflight to continue these investigations is scheduled for 2017. Finally, the study of droplets of immiscible materials on smectic bubbles is in its infancy. Of particular interest would be to investigate the motion of inclusions on films with non-uniform Gaussian curvature, such as catenoids or Delauney surfaces in general. In such experiments, gravitation generally masks all capillary effects in uniformly thick films. With a slight modification of the OASIS setup, all these studies would become feasible in microgravity.

\section{Acknowledgments}

The authors acknowledge financial support for the OASIS project from NASA Grant NNX-13AQ81G, and from the DLR within projects OASIS-Co 50WM1127 and 50WM1430. We are particularly indebted to the DLR for making the TEXUS and parabolic flights possible, and to Airbus DS for the construction and testing of the OASIS-TEx equipment, as well as for technical support during the TEXUS 52 campaign. ZIN Technologies, Inc. is acknowledged for constructing and testing the OASIS ISS equipment and for providing control of the ISS experiment during the mission. Drawings of the setup shown here were reproduced from ZIN design documents. The authors are grateful to Jennifer Storck and the ZIN engineers for their dedicated, professional support during the ISS mission. We are also indebted to Adam Green for helping to monitor the experiments during this long undertaking. Finally, we would like to thank the astronauts and cosmonauts for their enthusiastic engagement and for helping to make the OASIS mission a success. 


\section{References}

[1] C. Y. Young, R. Pindak, N. A. Clark, R. B. Meyer, Light-scattering study of two-dimensional molecular- orientation fluctuations in a freely suspended ferroelectric liquid crystal film, Phys. Rev. Lett. 40 (1978) 773.

[2] D. Van Winkle, N. Clark, Direct measurement of orientation correlations: observation of the landau-peierls divergence in a freely suspended tilted smectic film, Phys. Rev. Lett. 53 (1984) 1157.

[3] D. R. Link, A. Tewary, N. A. Clark, Compressionally induced smectic c to smectic a transitions in a freely suspended liquid crystal film, Bull. Am. Phys. Soc. 42 (1997) 382.

[4] T.Trittel, K. Harth, R. Stannarius, Smectic C to smectic A transition induced mechanically by the rupture of freely suspended liquid crystal films, Soft Matter in press.

[5] R. Pindak, C. Y. Young, R. B. Meyer, N. A. Clark, Macroscopic orientation patterns in smectic-c films, Phys. Rev. Lett. 45 (1980) 1193.

[6] R. Stannarius, K. Harth, Defect interactions in anisotropic two-dimensional fluids, Phys. Rev. Lett. 117 (2016) 157801.

[7] C. D. Muzny, N. A. Clark, Direct observation of the brownian motion of a liquid-crystal topological defect, Phys. Rev. Lett. 68 (1992) 804.

[8] M. Cheng, J. T. Ho, S. W. Hui, R. Pindak, Electron-diffraction study of free-standing liquid-crystal films, Phys. Rev. Lett. 59 (1987) 1112.

[9] R. Pindak, D. E. Moncton, S. C. Davey, J. W. Goodby, X-ray observation of a stacked hexatic liquid-crystal b phase, Phys. Rev. Lett. 46 (1981) 1135.

[10] J. M. Kosterlitz, D. J. Thouless, Ordering, metastability and phase transitions in two-dimensional systems, J. Phys. C: Solid State Phys. 6 (1973) 1181.

[11] Y. Iwashita, S. Herminghaus, R. Seemann, C. Bahr, Smectic membranes in aqueous environment, Phys. Rev. E 81 (2010) 051709.

[12] K. Harth, R. Stannarius, Measurement of the interface tension of smectic membranes in water, Phys. Chem. Chem. Phys. 15 (2013) 7204.

[13] P. Pieranski, L. Beliard, J.-P. Tournellec, X. Leoncini, C. Furtlehner, H. Dumoulin, E. Riou, B. Jouvin, J.-P. Fenerol, P. Palaric, J. Heuving, B. Cartier, I. Kraus, Physics of smectic membranes, Physica A 194 (1993) 364.

[14] P. Oswald, P. Pieranski, Smectic and Columnar Liquid Crystals: Concepts and Physical Properties Illustrated by Experiments, Taylor \& Francis, Boca Raton, 2005. 
[15] F. Picano, R. Hołyst, P. Oswald, Coupling between meniscus and smecticA films: Circular and catenoid profiles, induced stress, and dislocation dynamics, Phys. Rev. E 62 (2000) 3747.

[16] Z. Qi, C. S. Park, M. A. Glaser, J. E. Maclennan, N. A. Clark, Experimental realization of an incompressible newtonian fluid in two dimensions, Phys. Rev. E 93 (2016) 012706.

[17] K. Simons, E. Ikonen, Functional rafts in cell membranes, Nature (London) 387 (1997) 569.

[18] N. Oppenheimer, H. Diamant, Correlated diffusion of membrane proteins and their effect on membrane viscosity, Biophys. J. 96 (2009) 3041.

[19] P. G. Saffman, M. Delbrück, Brownian motion in biological membranes, Proc. Natl. Acad. Sci. USA 72 (1975) 3111.

[20] P. G. Saffman, Brownian motion in thin sheets of viscous fluid, J. Fluid Mech. 73 (1976) 593.

[21] A. J. Levine, T. B. Liverpool, F. C. MacKintosh, Mobility of extended bodies in viscous films and membranes, Phys. Rev. E 69 (2004) 021503. doi:10.1103/PhysRevE.69.021503

[22] E. Petrov, P. Schwille, Translational diffusion in lipid membranes beyond the saffman-delbrück approximation, Biophys. J. 94 (2008) L41.

[23] Z. H. Nguyen, M. Atkinson, C. S. Park, J. Maclennan, M. Glaser, N. Clark, Crossover between 2D and 3D fluid dynamics in diffusion of islands in ultrathin freely suspended smectic films, Phys. Rev. Lett. 105 (2010) 268304.

[24] A. Eremin, S. Baumgarten, K. Harth, R. Stannarius, Z. H. Nguyen, A. Goldfain, C. S. Park, J. E. Maclennan, M. A. Glaser, N. A. Clark, Twodimensional microrheology of freely suspended liquid crystal films, Phys. Rev. Lett. 107 (2011) 268301. doi:10.1103/PhysRevLett.107.268301.

[25] Z. Qi, Z. H. Nguyen, C. S. Park, M. A. Glaser, J. E. Maclennan, N. A. Clark, T. Kuriabova, T. R. Powers, Mutual diffusion of inclusions in freely suspended smectic liquid crystal films, Phys. Rev. Lett. 113 (2014) 128304.

[26] T. Kuriabova, T. R. Powers, Z. Qi, A. Goldfain, C. S. Park, M. A. Glaser, J. E. Maclennan, N. A. Clark, Hydrodynamic interactions in freely suspended liquid crystal films, Phys. Rev. E 94 (2016) 052701.

[27] P. E. Cladis, Y. Couder, H. R. Brand, Phase winding and flow-alignment in freely suspended films of smectic-C liquid crystals, Phys. Rev. Lett. 55 (1985) 2945.

[28] P. E. Cladis, W. Van Saarloos, Solitons in Liquid Crystals, SpringerVerlag New York Berlin Heidelberg, 1992, Ch. Some Nonlinear Problems in Anisotropic Systems, p. 110. 
[29] P. E. Cladis, P. L. Finn, H. R. Brand, Stable coexistence of spiral and target patterns in freely suspended films of smectic-C liquid crystals, Phys. Rev. Lett. 75 (1995) 1518.

[30] C. Chevallard, J. M. Gilli, T. Frisch, I. V. Chikina, P. Pieranski, Cladis' orbiting disclinations in smectic films submitted to a torque, Mol. Cryst. Liq. Cryst. 328 (1999) 589-594.

[31] C. Chevallard, J. M. Gilli, T. Frisch, I. V. Chikina, P. Pieranski, "magic spiral" submitted to a torque: Topological flows driven by ericksen stresses in sm c films, Mol. Cryst. Liq. Cryst. 328 (1999) 595-611.

[32] D. R. Link, L. Radzihovsky, G. Natale, J. E. Maclennan, Ring-pattern dynamics in smectic- $\mathrm{C}^{*}$ and smectic-C- $\mathrm{A}^{*}$ freely suspended liquid crystal films, Phys. Rev. Lett 84 (2000) 5772.

[33] R. Stannarius, C. Bohley, A. Eremin, Vortex flow in free standing smectic films driven by elastic relaxation of the c-director, Phys. Rev. Lett. 97 (2006) 97802.

[34] I. Mutabazi, P. L. Finn, J. T. Gleeson, J. W. Goodby, C. D. Andereck, P. E. Cladis, Ring pattern-formation in 2-d shear of freely suspended films of a smectic-C liquid crystal, Europhys. Lett. 19 (1992) 391-395.

[35] M. I. Godfrey, D. H. Van Winkle, Surface-tension-gradient-induced flow in freely suspended liquid crystalline films, Phys. Rev. E. 54 (1996) 3752-3764.

[36] J. Birnstock, R. Stannarius, Vertically suspended smectic films with inplane temperature gradients, Mol. Cryst. Liq. Cryst. 366 (2001) 815.

[37] S. W. Morris, J. R. De Bruyn, A. D. May, Electroconvection and patternformation in a suspended smectic film, Phys. Rev. Lett. 65 (1990) 2378.

[38] S. W. Morris, J. R. De Bruyn, A. D. May, Velocity and current measurements in electroconvecting smectic films, Phys. Rev. A 44 (1991) 8146-8157.

[39] C. Langer, R. Stannarius, Electroconvection in freely suspended smectic-C and smectic-C* films, Phys. Rev. E. 58 (1998) 650.

[40] M. Conradi, P. Ziherl, A. Sarlah, I. Musevic, Colloids on free-standing smectic films, Eur. Phys. J. E. 20 (2006) 231-236.

[41] K. Meienberg, T. Malinina, P. C. G. M. C. N. M. J. Nguyen, Z., Nanoparticle aggregation and fractal growth in fluid smectic membranes, Mol. Cryst. Liq. Cryst 611 (2015) 14-20.

[42] C. Bohley, R. Stannarius, Inclusions in free standing smectic liquid crystal films, Soft Matter 4 (2008) 683.

[43] P. Cluzeau, P. Poulin, G. Joly, H. T. Nguyen, Two-dimensional ordering of inclusions in smectic C phase, Phys. Rev. E. 63 (2001) 031702. 
[44] P. Cluzeau, G. Joly, H. T. Nguyen, V. K. Dolganov, Two-dimensional ordering of inclusions in smectic C phase, JETP Letters 75 (2002) 482-486.

[45] P. Cluzeau, G. Joly, H. T. Nguyen, V. K. Dolganov, Formation of twodimensional crystal-like structures from inclusions in smectic C films, JETP Letters 76 (2002) 351-354.

[46] P. Cluzeau, P. Bonnand, G. Joly, V. K. Dolganov, H. T. Nguyen, Selforganization of $\mathrm{N}^{*}$ inclusions in $\mathrm{SmC}^{*}$ free-standing films, Eur. Phys. J. E.. 10 (2003) 231-240.

[47] C. Völtz, R. Stannarius, Self-organization of isotropic droplets in smectic-C free-standing films, Phys. Rev. E. 70 (2004) 061702.

[48] C. Völtz, R. Stannarius, Buckling instability of droplet chains in freely suspended smectic films, Phys. Rev. E. 72 (2005) 011705.

[49] K. May, K. Harth, T. Trittel, R. Stannarius, Dynamics of freely floating smectic bubbles, Europhys. Lett. 100 (2014) 16003.

[50] K. May, K. Harth, T. Trittel, R. Stannarius, Freely floating smectic films, Chem. Phys. Chem. 15 (2014) 1508.

[51] P. P. P. F. Perrin, J., R. Holyst, When boundaries dominate: dislocation dynamics in smectic films., Phys. Rev. Lett. 88 (2002) 015503.

[52] R. Stannarius, C. Cramer, Self-supporting bubbles of thermotropic smectic liquid crystals, Europhys. Lett. 42 (1998) 43.

[53] F. Müller, R. Stannarius, Collapse of catenoid-shaped smectic films, Europhys. Lett. 76 (2006) 1102.

[54] R. Stannarius, T. Trittel, A. Eremin, K. Harth, C. Park, M. Glaser, N. Clark, J. Maclennan, Liquid crystal films and bridges in microgravity, Proceedings of 22nd ESA Symposium on European Rocket and Balloon Programmes and Related Research (Troms $\varnothing$, Norway).

[55] S. Dölle, K. Harth, T. John, R. Stannarius, Impact and embedding of picoliter droplets into freely suspended smectic films, Langmuir 30 (2014) 12712 .

[56] H. Schüring, R. Stannarius, Isotropic droplets in thin free standing smectic films, Langmuir 18 (2002) 9735-9743.

[57] H. Schüring, R. Stannarius, Surface and interface tensions determined from isotropic droplets in freely suspended smectic films, Mol. Cryst. Liq. Cryst. 412 (2004) 2035. 